\title{
Intramolecular coupling as a mechanism for a liquid-liquid phase transition
}

\author{
Giancarlo Franzese, ${ }^{1,2, *}$ Manuel I. Marqués, ${ }^{1}$ and H. Eugene Stanley ${ }^{1}$ \\ ${ }^{1}$ Center for Polymer Studies and Department of Physics, Boston University, Boston, Massachusetts 02215 \\ ${ }^{2}$ Dipartimento di Ingegneria dell'Informazione, Seconda Università di Napoli, INFM UdR Napoli and CG SUN, \\ via Roma 29, I-81031 Aversa (CE), Italy
}

(Received 11 March 2002; published 22 January 2003)

\begin{abstract}
We study a model for water with a tunable intramolecular interaction $J_{\sigma}$, using mean-field theory and off-lattice Monte Carlo simulations. For all $J_{\sigma} \geqslant 0$, the model displays a temperature of maximum density. For a finite intramolecular interaction $J_{\sigma}>0$, our calculations support the presence of a liquid-liquid phase transition with a possible liquid-liquid critical point for water, likely preempted by inevitable freezing. For $J$ $=0$, the liquid-liquid critical point disappears at $T=0$.
\end{abstract}

DOI: 10.1103/PhysRevE.67.011103

PACS number(s): 64.70.Ja, 05.10.Ln, 05.70.Ce

\section{INTRODUCTION}

Water has an anomalous density decrease for isobaric cooling below a temperature of maximum density (TMD) [1]. Other thermodynamic anomalies — such as the rapid increase of the response functions - can be fit by power laws with apparent singularity well below the freezing temperature [1]. Several interpretations for this behavior have been proposed, but it is unclear which describes water or if any of them describes other anomalous liquids including, among the others, $\mathrm{S}, \mathrm{Se}, \mathrm{Te}, \mathrm{Cs}, \mathrm{Si}, \mathrm{Ge}, \mathrm{I}, \mathrm{C}, \mathrm{P}, \mathrm{SiO}_{2}$, and $\mathrm{BeF}_{2}$ [2-18].

One of the interpretations, the stability-limit conjecture [19], assumes that the limits of stability of the superheated, supercooled, and stretched liquid form a single retracing spinodal line in the pressure-temperature $(P-T)$ plane. This scenario predicts a divergence of the response functions at the supercooled liquid-to-liquid spinodal [20].

The singularity-free interpretation [21,22] envisages that the experimental data represent apparent singularities, due to anticorrelated fluctuations of volume and entropy. In this scenario, these fluctuations are responsible for the TMD line.

The liquid-liquid phase transition hypothesis [23] proposes the presence of a first-order line of phase transitions separating two liquid phases differing in density, the highdensity liquid (HDL) and the low-density liquid (LDL). In this scenario the HDL-LDL phase transition, possibly ending in a liquid-liquid critical point, is responsible for the anomalies.

Although Refs. [8,9], by tuning parameters of the corresponding models, predict smooth transitions from the different scenarios, to help elucidate which is the most reasonable description for water, we consider a model fluid with intermolecular and intramolecular interactions. This model, in the particular case of a zero intramolecular interaction, recovers the model introduced by Sastry et al. [22], which predicts the singularity-free scenario. Our aim is to understand how the presence of an intramolecular interaction changes this prediction.

We perform analytic calculations in a mean-field approxi-

\footnotetext{
*Present address: SMC-INFM, Dipartimento di Fisica, Università “La Sapienza," P.le A. Moro 2, I-00185 Roma, Italy.
}

mation and an off-lattice Monte Carlo (MC) simulation. Our results show that a nonzero intramolecular interaction gives rise to a HDL-LDL phase transition, with a possible critical point [23], with the liquid-liquid critical temperature decreasing to zero and vanishing with the intramolecular interaction. Therefore, at least for this model, the singularity-free scenario is obtained only in the particular case of a zero intramolecular interaction, while for a finite intramolecular interaction the HDL-LDL phase transition is predicted. General considerations suggest that the liquid-liquid phase transition for water could occur below the glass temperature, i.e., outside the accessible experimental range.

The paper is organized as follows. In Sec. II, we define the model defined on a lattice. In Sec. III, we describe the equation of state approach in the mean-field approximation, and present the mean-field results. In Sec. IV we introduce the off-lattice model, describe the MC approach, and show the simulation results. In Sec. V, we discuss our results and give the conclusions.

\section{THE LATTICE MODEL}

The fluid is represented by partitioning the system into $N$ cells of equal size. A variable $n_{i}$ is associated with each cell $i=1, \ldots, N$, with $n_{i}=1$ if the cell is occupied by a molecule, $n_{i}=0$ otherwise.

The intermolecular interaction [22]

$$
\mathcal{H} \equiv-\epsilon \sum_{\langle i, j\rangle} n_{i} n_{j}-J \sum_{\langle i, j\rangle} n_{i} n_{j} \delta_{\sigma_{i j}, \sigma_{j i}}
$$

has a first term describing the van der Waals attraction between molecules, where $\epsilon>0$ is the energy gain for two nearest neighbor (nn) occupied cells and the sum is over all the possible nn cells.

The second term in Eq. (1) accounts for the dynamic network of hydrogen bonds (HBs) formed by liquid water, with each molecule typically bonded to four other molecules at low $T$ [1], with an energy gain $J>0$ per HB. We consider cells with size of a water molecule and with four arms, one per possible HB. For the molecule in the cell $i$, the orientation of the arm facing the cell $j$ is represented by a Potts variable $\sigma_{i j}=1, \ldots, q$, with a finite number $q$ of possible 
orientations. Two molecules in nn cells form a HB only if they are correctly oriented [1], i.e., by assumption [22], if $\delta_{\sigma_{i j}, \sigma_{j i}}=1 \quad\left(\delta_{a, b}=1\right.$ if $a=b$ and $\delta_{a, b}=0$ otherwise).

The experimental oxygen-oxygen correlation function shows that a HB is formed if and only if the intermolecular distance is within a characteristic range [1]. Hence, we assume that the formation of a HB leads to a local volume expansion [22]

$$
V \equiv V_{0}+N_{H B} v_{H B},
$$

where $V_{0}$ is the volume of the liquid with no HBs;

$$
N_{H B} \equiv \sum_{\langle i, j\rangle} n_{i} n_{j} \delta_{\sigma_{i j}, \sigma_{j i}}
$$

is the total number of HBs in the system, and $v_{H B}$ is the specific volume per HB.

Experiments show that the relative orientations of the arms of a water molecule are correlated, with the average $\mathrm{H}-\mathrm{O}-\mathrm{H}$ angle equal to $104.45^{\circ}$ in an isolated molecule, $104.474^{\circ}$ in the gas, and $106^{\circ}$ in the high- $T$ liquid [24], suggesting an intramolecular interaction between the arms. This interaction must be finite, because the angle changes with $T$, consistent with $a b$ initio calculations [25] and molecular dynamics simulations [26]. Hence, we introduce the intramolecular (IM) term $[27,28]$

$$
\mathcal{H}_{I M} \equiv-J_{\sigma} \sum_{i} n_{i} \sum_{(k, l)_{i}} \delta_{\sigma_{i k}, \sigma_{i l}}
$$

where for each of the ${ }^{4} C_{2}=6$ different pairs $(k, l)_{i}$ of the arms of a molecule $i$, with the appropriate orientation $\left(\delta_{\sigma_{i k}, \sigma_{i l}}=1\right)$, there is an energy gain $J_{\sigma}>0$.

For $J_{\sigma}=0$, we recover the model of Ref. [22], which predicts the singularity-free scenario, and where the HBs are uncorrelated, inhibiting the orientational long-range order. We study the general case with finite $J_{\sigma}$, by using (i) a mean field approximation and (ii) MC simulations.

\section{THE EQUATION OF STATE APPROACH}

The equation of state of our system is implicitly given by

$$
U-T S+P V=\mu \sum_{i} n_{i},
$$

where

$$
U \equiv \mathcal{H}+\mathcal{H}_{I M}
$$

is the total internal energy and $\mu$ is the chemical potential. From Eqs. (1)-(4), we rewrite the equation of state as

$$
T S-P V_{0}=-\sum_{\langle i, j\rangle} \epsilon_{i j}^{\prime}(P, \sigma) n_{i} n_{j}-\sum_{i} \mu_{i}^{\prime}(\sigma) n_{i} .
$$

Here

$$
\epsilon_{i j}^{\prime}(P, \sigma) \equiv \epsilon+J^{\prime}(P) \delta_{\sigma_{i j}, \sigma_{j i}}
$$

is the effective attraction energy, depending on $P$ and the local arm configuration,

$$
J^{\prime}(P) \equiv J-P v_{H B}
$$

is the effective HB interaction energy due to the HB volume increase, and

$$
\mu_{i}^{\prime}(\sigma) \equiv \mu+J_{\sigma} \sum_{(k, l)} \delta_{\sigma_{k i}, \sigma_{l i}}
$$

is the effective local chemical potential depending on the local arm configuration.

\section{A. The mean-field approximation}

The mean-field approximation consists in assuming a linear relation between the number density of liquid cells,

$$
n \equiv \frac{1}{N} \sum_{i} n_{i},
$$

and the density order parameter $m \in[-1,1]$, and between the number density of arms in the appropriate state for a HB,

$$
n_{\sigma} \equiv \sum_{\langle i, j\rangle} \delta_{\sigma_{i j}, 1},
$$

and the orientational order parameter $m_{\sigma} \in[0,1]$, i.e.,

$$
n=\frac{1+m}{2}, n_{\sigma}=\frac{1+(q-1) m_{\sigma}}{q} .
$$

Hence, the molar density $\rho \equiv n N / V$ is

$$
\rho=\frac{1+m}{2 v_{0}+4 v_{H B} p_{H B}} .
$$

Here $v_{0} \equiv V_{0} / N$, and $p_{H B} \equiv n^{2} p_{\sigma}$ is the probability of forming a HB between two nn molecules, where $n^{2}$ is the probability of finding two nn molecules, and

$$
p_{\sigma} \equiv n_{\sigma}^{2}+(q-1)\left(\frac{1-n_{\sigma}}{q-1}\right)^{2}=\frac{1+(q-1) m_{\sigma}^{2}}{q}
$$

is the probability of having the facing arms of the two molecules in the appropriate orientational state for a HB.

For $T \rightarrow \infty$ we expect $m_{\sigma} \rightarrow 0$, hence $p_{\sigma} \rightarrow 1 / q$. For $T$ $\rightarrow 0$, the finite values of $\epsilon, J$, and $J_{\sigma}$ allow us to assume a cooperative effect and an orientational long-range order in a preferred state, with $m_{\sigma} \rightarrow 1$, hence $p_{\sigma} \rightarrow 1$.

\section{B. The cooperative effect}

To include the cooperative effect, we consider that each arm $\sigma_{i j}$ interacts with a mean field $h$ generated by the other three arms on the same molecule, in addition to the effective interaction $J^{\prime}(P)$ with the facing arm $\sigma_{j i}$ on a nn molecule. Since the energy is minimized when the arms are in the same orientational state, the system breaks the symmetry ordering 
in the preferred Potts state. Hence, we choose $h$ proportional to $n_{\sigma}$, to $J_{\sigma}$, and to the number of arms generating $h$, i.e.,

$$
h \equiv 3 J_{\sigma} n_{\sigma}
$$

Our results do not depend on this choice for $h$, and are recovered using higher-order approximations for $h$.

We assume

$$
p_{\sigma}=\left\langle\delta_{\sigma_{i j}, \sigma_{j i}}\right\rangle_{h},
$$

i.e., we assume that $p_{\sigma}$ [Eq. (15)], for two nn molecules interacting with the surrounding, coincides with the probability $\left\langle\delta_{\sigma_{i j}, \sigma_{j i}}\right\rangle_{h}$, for the facing arms $\left(\sigma_{i j}, \sigma_{j i}\right)$ of two isolated $\mathrm{nn}$ molecules, of being in the same orientational state under the action of the field $h$. By definition,

$$
\begin{aligned}
\left\langle\delta_{\sigma_{i j}, \sigma_{j i}}\right\rangle_{h} \equiv & \frac{1}{\mathcal{Z}_{h}} \sum_{\sigma_{i j}, \sigma_{j i}} \delta_{\sigma_{i j}, \sigma_{j i}} \exp \left\{\left[J^{\prime}(P) \delta_{\sigma_{i j}, \sigma_{j i}}\right.\right. \\
& \left.\left.+h\left(\delta_{\sigma_{i j}, 1}+\delta_{\sigma_{j i}, 1}\right)\right] /\left(k_{B} T\right)\right\} \\
= & {\left[1+(q-1) \frac{2 w_{m_{\sigma}}+q-2}{w\left(w_{m_{\sigma}}^{2}+q-1\right)}\right]^{-1}, }
\end{aligned}
$$

where the rightmost side is the explicit calculation of the left side with partition function $\mathcal{Z}_{h}$. Here the sum is over all the configurations of the two variables $\sigma_{i j}, \sigma_{j i}$, and the symbols are

$$
\begin{gathered}
w \equiv \exp \left[\frac{J^{\prime}(P)}{k_{B} T}\right], \\
w_{m_{\sigma}} \equiv \exp \left\{\frac{3 J_{\sigma}\left[1+m_{\sigma}(q-1)\right]}{q k_{B} T}\right\}, \\
\mathcal{Z}_{h} \equiv \sum_{\sigma_{i j}, \sigma_{j i}} \exp \left[\frac{J^{\prime}(P) \delta_{\sigma_{i j}, \sigma_{j i}}+h\left(\delta_{\sigma_{i j}, 1}+\delta_{\sigma_{j i}, 1}\right)}{k_{B} T}\right],
\end{gathered}
$$

with the Boltzmann constant $k_{B}$ chosen as unitary hereafter.

As expected for $p_{\sigma}$, also $\left\langle\delta_{\sigma_{i j}, \sigma_{j i}}\right\rangle_{h} \rightarrow 1 / q$ for $T \rightarrow \infty$ and $\left\langle\delta_{\sigma_{i j}, \sigma_{j i}}\right\rangle_{h} \rightarrow 1$ for $T \rightarrow 0$. Numerically we find that the solution of Eq. (17) is $m_{\sigma}^{*}(T, P)=0$ for $P>P_{\max }(T)$, and $m_{\sigma}^{*}(T, P)>0$ for $P \leqslant P_{\text {max }}(T)$, where $m_{\sigma}^{*}(T, P)=0$ corresponds to the lack of orientational order and $m_{\sigma}^{*}(T, P)>0$ corresponds to the orientational long-range order. In this mean-field approximation, $P_{\max }(T)$ turns out to be well described by a decreasing linear function of $T$.

\section{The Gibbs free energy}

Next, we write a mean-field expression for the molar Gibbs free energy

$$
g \equiv u-T s+P v=\mu
$$

as a function of the two order parameters $m$ and $m_{\sigma}^{*}$, where

$$
u \equiv \frac{U}{n N}=-2\left[\epsilon n+\left(J n+3 J_{\sigma}\right) p_{\sigma}\right]
$$

is the molar energy as derived by Eqs. (1)-(4) and Eq. (6), with the mean-field approximations

$$
\begin{gathered}
\sum_{\langle i, j\rangle} n_{i} n_{j}=n^{2}, \\
\sum_{\langle i, j\rangle} n_{i} n_{j} \delta_{\sigma_{i j}, \sigma_{j i}}=n^{2} p_{\sigma}, \\
\sum_{i} n_{i} \sum_{(k, l)_{i}} \delta_{\sigma_{i k}, \sigma_{i l}}=n p_{\sigma} ;
\end{gathered}
$$

$v \equiv 1 / \rho$ is the molar volume derived by Eq. (14);

$$
s \equiv \frac{S_{W}+S_{\sigma}}{n N}
$$

is the molar entropy,

$$
\begin{gathered}
-\frac{S_{W}}{k_{B} N}=n \ln n+(1-n) \ln (1-n), \\
-\frac{S_{\sigma}}{4 k_{B} n N}=n_{\sigma}^{*} \ln n_{\sigma}^{*}+(q-1)\left(\frac{1-n_{\sigma}^{*}}{q-1}\right) \ln \left(\frac{1-n_{\sigma}^{*}}{q-1}\right)
\end{gathered}
$$

are the standard mean-field expressions for the entropy of $N$ variables $\left(n_{i}\right)$ and for the entropy of $4 n N q$-state Potts variables for the arms, respectively, and $n_{\sigma}^{*}$ is the number density $n_{\sigma}$ of H-bonded arms [Eq. (13)] evaluated in $m_{\sigma}^{*}(T, P)$.

\section{The mean-field results}

By numerically minimizing $g(T, P)$ with respect to $m$ and $m_{\sigma}^{*}$ with the constraint that $m_{\sigma}^{*}$ is solution of Eq. (17), we find the equilibrium values of $m(T, P)$ and $m_{\sigma}^{*}(T, P)$. By using Eq. (14), we find $\rho(T, P)$ at equilibrium (Fig. 1).

At high $P$, the mean-field theory predicts that $\rho(T)$ increases when $T$ decreases (Fig. 1). At low $P$, for decreasing $T$, the theory predicts (i) a discontinuity in $\rho(T)$, corresponding to the liquid-gas first-order phase transition ending in the liquid-gas critical point $C$ (Fig. 2); (ii) decreasing TMD with increasing $P$; and (iii) a discontinuity in $\rho(T)$ at low $T$, disappearing at lower $P$.

The first two predictions are consistent with either the singularity-free scenario or the liquid-liquid phase transition hypothesis, while the third prediction is consistent only with the HDL-LDL first-order phase transition hypothesized in the latter scenario. In particular, the smooth disappearing of the discontinuity at lower $P$ is consistent with a phase transition line, with a negative slope in the $P-T$ phase diagram, ending in a HDL-LDL critical point $C^{\prime}$ (Fig. 2).

\section{THE OFF-LATTICE MODEL}

To show that our mean-field predictions are robust, we now use a completely different approach based on an off- 


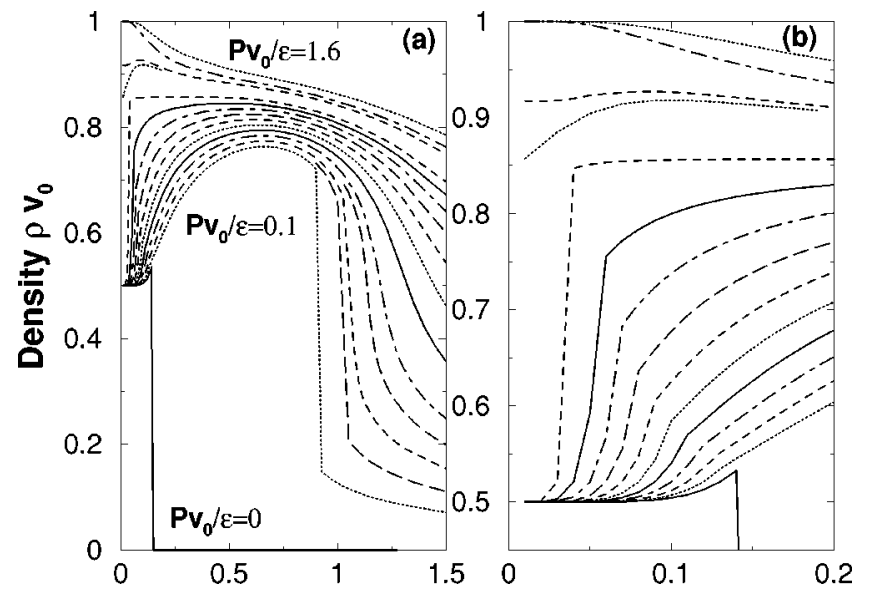

FIG. 1. The mean-field isobaric molar density $\rho$ as function of $T$ for (top to bottom) $P v_{0} / \epsilon=1.6,1.4,1.3,1.275,1.0,0.9,0.8,0.7$, $0.6,0.5,0.4,0.3,0.25,0.2,0.15,0.1,0$, for the model with parameters $q=6, J / \epsilon=0.5, J_{\sigma} / \epsilon=0.05$, and $v_{H B} / v_{0}=0.5$. (a) For 0.1 $\lesssim P v_{0} / \epsilon \lesssim 0.25$, by decreasing $T, \rho$ has a discontinuity at high $T$ [from a low value (in the gas phase) to a high value (in the liquid phase)], then $\rho$ has a maximum followed by a smooth saturation to the finite value $\rho_{H B}=0.5 / v_{0}$ corresponding to the full-H-bonded liquid. For $0.3 \leqq P v_{0} / \epsilon \lesssim 0.4$, by decreasing $T$, there is no discontinuity in $\rho$, but there is a maximum in $\rho$ and the saturation to $\rho_{H B}$. For $0.5 \leqq P v_{0} / \epsilon \lesssim 1.2$, by decreasing $T, \rho$ has a maximum and then a discontinuity to $\rho_{H B}$. For $1.25 \leqq P v_{0} / \epsilon \lesssim 1.6, \rho$ has only a maximum, and for higher $P, \rho$ regularly increases by decreasing $T$. (b) Blowup of the low- $T$ region. Both discontinuities reported show a first-order phase transition, each ending in a critical point (Fig. 2).

lattice (OL) model representing a system with a homogeneous distribution of molecules in the available volume $V$, which we divide in $N$ equivalent cells of volume $V / N$.

As a consequence of the homogeneity of the system, for each cell, the $N$ degrees of occupancy freedom $\left(n_{i}\right)$ are set to $n_{i}=1$. In analogy with Eq. (2), the total volume $V$ is defined as

$$
V \equiv V_{0}^{O L}+N_{H B} v_{H B}
$$

Here $N_{H B}$ and $v_{H B}$ are defined as in Eqs. (2) and (3), but, different from the lattice case, the volume $V_{0}^{O L}$ associated with the total volume of the cells without HBs is a continuous variable with the constrains $V_{0}^{O L} \geqslant N v_{0}$, where $v_{0}$ is the hard-core volume of a molecule.

Also, following the lattice model, the molecules have four arms described by four $q$-state variables $\sigma_{i j}$, with the HB interaction defined by the second term in Eq. (1) and the intramolecular interaction by Eq. (4). These interactions are both independent of the distance among first-neighbor molecules, and depend only on the arms orientation $\sigma_{i j}$.

In this off-lattice model, the average distance between two molecules $(r)$ is a continuous variable, so we replace the first term in Eq. (1) with

$$
U_{W}(r) \equiv\left\{\begin{array}{l}
\infty \quad \text { for } r \leqslant R_{0} \\
\epsilon\left[\left(\frac{R_{0}}{r}\right)^{12}-\left(\frac{R_{0}}{r}\right)^{6}\right] \quad \text { for } r>R_{0},
\end{array}\right.
$$

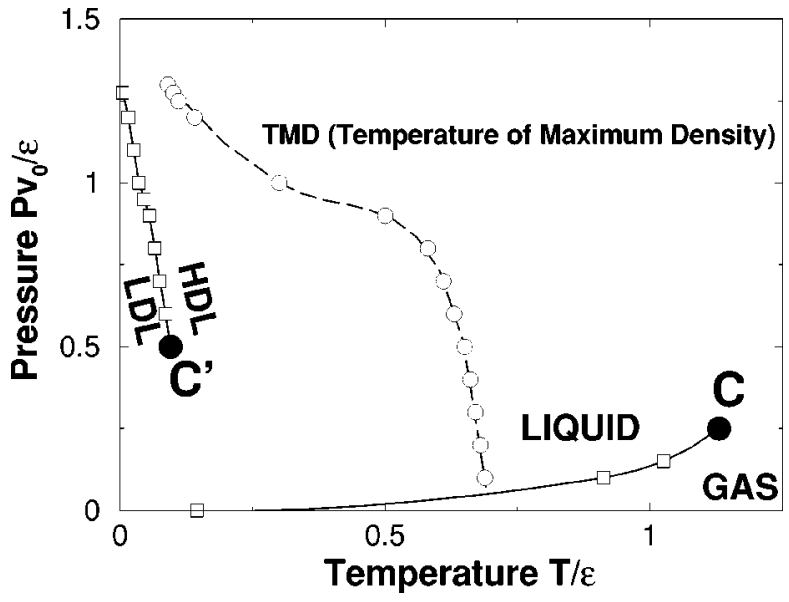

FIG. 2. The $P-T$ phase diagram for the model with the parameters in Fig. 1. The squares and the circles are estimated from the discontinuities and the maxima in Fig. 1, respectively. Since the symmetry between the two phases separated by a critical point must be preserved, the high- $T$ discontinuity shows a gas-liquid phase transition, while the low- $T$ discontinuity shows the HDL-LDL phase transition. The lines are guides for the eyes. The liquid-gas phase transition line ends in the critical point $C$. The HDL-LDL phase transition line, with negative slope, ends in the critical point $C^{\prime}$. The dashed line corresponds to the TMD line.

where $R_{0} \equiv \sqrt{v_{0}}$ is the hard-core diameter of each molecule. In analogy with the Eq. (1), we consider this off-lattice van der Waals energy independent of the HB expansion, so in $U_{W}(r)$, we use

$$
r \equiv \sqrt{V_{0}^{O L} / N}
$$

\section{The Monte Carlo simulation}

We perform MC simulations, in two dimensions [29], at constant $N, P$, and $T$, and variable $V(N-P-T$ ensemble) with $N \in\left[10^{2}, 10^{4}\right]$. The MC dynamics consists in updating the variables $\sigma_{i j}$ and the variable $V_{0}^{O L}$, accepting the new state with probability $\exp \left[-\left(\Delta U_{W}+P \Delta V / k_{B} T\right)\right]$ if $\Delta U+P \Delta V>0$, or with probability 1 if $\Delta U+P \Delta V<0$. Here $\Delta U \equiv \Delta\left(U_{W}\right.$ $\left.+\mathcal{H}_{I M}\right)$, and $\Delta V$ are the changes of total internal energy and total volume, Eq. (25), respectively, after the update. Our results for the average density $\rho^{M C} \equiv N / V$, averaged over $6 \times 10^{5} \mathrm{MC}$ steps after $1.2 \times 10^{5} \mathrm{MC}$ steps of thermalization at each $T$, are qualitatively consistent with the mean-field prediction (Fig. 3).

By MC simulations, we find (i) for $J_{\sigma}=0$, no liquidliquid phase transition and the TMD line, consistent with mean-field in Ref. [22]; (ii) for $J_{\sigma} \gg J$, for any $P$ in the liquid phase, at $T$ below the TMD line, a discontinuity in density, suggesting a first-order phase transition along a line with a negative slope in the $P-T$ phase diagram, consistent with mean field for $J_{\sigma} \rightarrow \infty[27,28]$; (iii) for $0<J_{\sigma}<J$, a phase transition line ending in a critical point $C^{\prime}$ (Fig. 4), and occurring at increasing $P$ and decreasing $T$ for decreasing $J_{\sigma}$.

To verify that the jumps found in the MC density are marking a first-order phase transition, instead of a narrow 


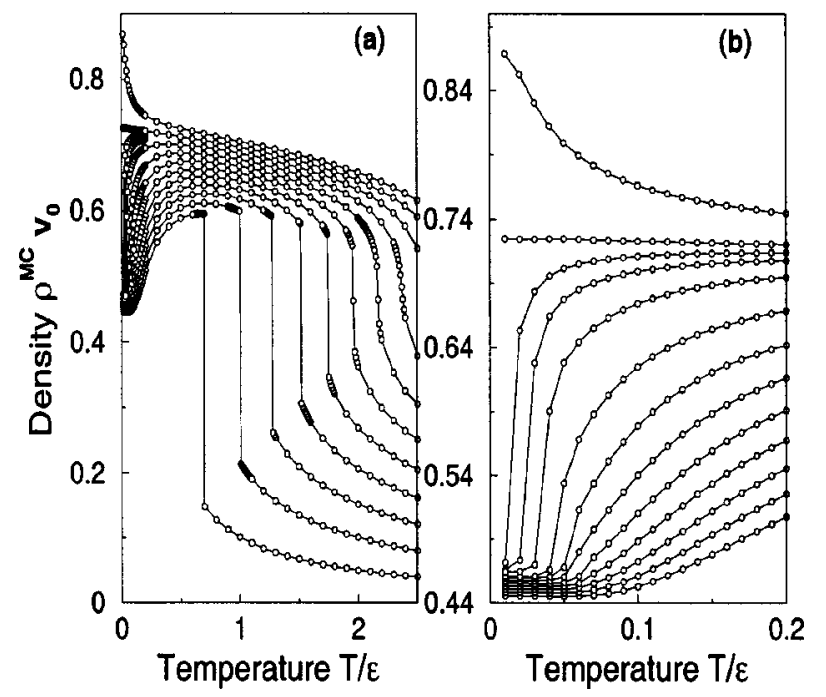

FIG. 3. MC isobaric density $\rho^{M C}(T)$ for $N=10^{4}$ molecules, for the off-lattice model with parameters as in Fig. 1. We show only the isobars for (bottom to top) $P v_{0} / \epsilon=0.1,0.2,0.3,0.4,0.5,0.6,0.7,0.8$, $0.9,0.95,0.975,1,1.1$. (a) The qualitative behavior is as described in Fig. 1. (b) Blowup of the low- $T$ region.

continuous phase transition, we study the isothermal compressibility

$$
K_{T} \equiv-\frac{1}{V}\left(\frac{\partial V}{\partial P}\right)_{T}
$$

Its maximum $K_{T}^{\max }$, where

$$
\begin{gathered}
\left.\left(\partial K_{T} / \partial V\right)\right|_{T}=0, \\
\left.\left(\partial^{2} K_{T} / \partial V^{2}\right)\right|_{T}>0,
\end{gathered}
$$

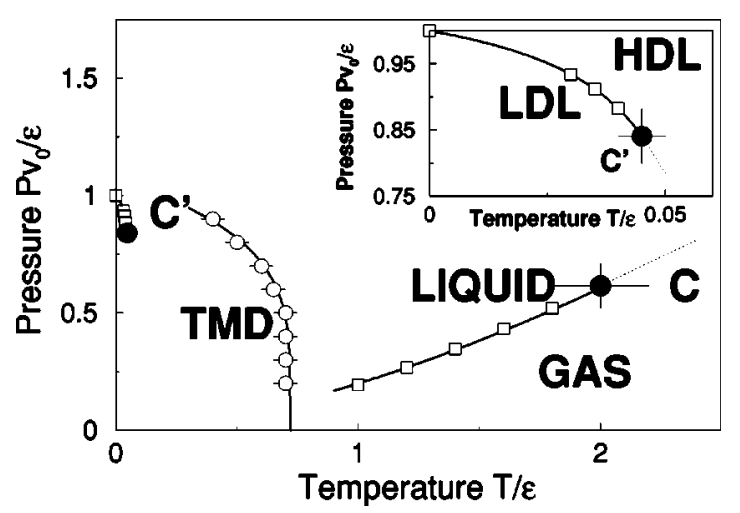

FIG. 4. $P-T$ phase diagram calculated by MC simulations, for the off-lattice model as in Fig. 3. Squares are the $N \rightarrow \infty$ estimates for the points on the coexisting lines. Full circles are the critical points $C$ and $C^{\prime}$. Points on the TMD line (open circles) are estimated from the $N \rightarrow \infty$ extrapolation of the maxima of $\rho^{M C}(T, N)$. Dashed lines indicate the position of $K_{T}^{\max }$ emanating from $C$ and $C^{\prime}$. Full lines are guides for the eyes. Where not shown, errors are smaller than the symbol size. Inset: blowup of the HDL-LDL phase transition region. The full line is given by the empirical expression $P_{\text {max }}=P_{0}-\left[a /\left(T_{0}-T\right)\right]$, where $P_{0} v_{0} / \epsilon=1.087, a v_{0} / \epsilon^{2}=0.006$, and $T_{0} / \epsilon=0.07$. increases linearly with the number of particles $N$ at a firstorder phase transition [30], while at a second order phase transition $K_{T}^{\max }$ is proportional to both $N$ and the fluctuation of the ordering parameter, scaling as a power of $N$ [31]. Therefore, the finite size scaling analysis on $K_{T}^{\max }(N)$ allows us to discriminate between a continuous and a discontinuous phase transition.

To estimate the maximum with a great precision, we use a continuous $T$ algorithm, the histogram reweighting method [32]. By checking the minimum $T$ and the maximum $P$ where the behavior of $K_{T}^{\max }(N)$ fails to be linear, we estimate the critical point at $T_{C^{\prime}} / \epsilon=0.045 \pm 0.005$ and $P_{C^{\prime}} v_{0} / \epsilon$ $=0.841 \pm 0.042$ for $N \rightarrow \infty$ (inset of Fig. 4).

Next, we obtain the coexisting lines by extrapolating to $N \rightarrow \infty$ the values $P(T, N)$ corresponding to $K_{T}^{\max }(N)$ at fixed $T$, both for $T \leqslant T_{C}$ and $T \leqslant T_{C^{\prime}}$. Furthermore, our results are consistent with the necessary condition that the $K_{T}^{\max }(T)$ line emanates from the critical point $[22,33]$, both for $T>T_{C^{\prime}}$ and for $T>T_{C}$ (Fig. 4).

\section{DISCUSSION AND CONCLUSIONS}

The mean-field and the MC results for our waterlike fluid model for a finite intramolecular interaction $J_{\sigma}$ show qualitatively the same phase diagram. In both approaches the TMD line decreases with increasing $P$, consistent with the experiments [4]. In particular, both approaches predict a first-order phase transition in the liquid phase, occurring at low $T$ and at a pressure $P_{\max }(T)$ decreasing for increasing $T$. For $J_{\sigma}<J$, we find that the first-order phase transition line ends in a critical point, separating (by necessity) two phases with the same symmetry, in this case two liquids (HDL and LDL) being the critical point in the liquid phase. Three considerations are in order here.

The first consideration is related to the comparison with the result of Ref. [22], here recovered for $J_{\sigma}=0$. The Sastry et al. model [22], upon HB formation, accounts for (i) intermolecular orientational correlation [Eq. (1)]; (ii) local expansion with lowering temperature [Eq. (2)]; (iii) anticorrelation between $V$ and $S$, because the formation of HBs decreases the number of possible orientational configurations for the system, hence the entropy $S$ decreases for increasing $N_{H B}$, i.e., for increasing $V$. This is expected in a system with a density anomaly, because $(\partial V / \partial T)_{P}<0$ implies $(\partial S / \partial V)_{T}$ $<0$. Finally, the Sastry et al. model assumes the arms of a molecule completely independent $\left(J_{\sigma}=0\right)$, and predicts the singularity-free scenario.

We tested, by preliminary MC calculations, that for $J_{\sigma}$ $\rightarrow 0$ the HDL-LDL critical point $C^{\prime}$ moves to lower $T$ and to $P_{\text {max }}(T=0)$. In particular, the phase transition disappears at $T=0$ for $J_{\sigma}=0$, while the effect of the decreasing $J_{\sigma}$ on the location of the TMD line is weak. Although these results require a longer analysis, beyond the scope of the present work, they show that the predictions of Ref. [22] are recovered in the limit $J_{\sigma}=0$, confirming the validity of our MC approach [34]. We, therefore, conclude that in this model the presence of a finite $J_{\sigma}$ is responsible for the appearance of the first-order phase transition, with a possible HDL-LDL critical point $C^{\prime}$. 
TABLE I. Characteristic temperatures and pressures for real water and for the present model, for the gas-liquid critical point $\left(T_{C}, P_{C}\right)$, the TMD at ambient pressure $\left(T^{*}, P^{*}\right)$, and the glass temperature $T_{g}$ at ambient pressure. The ratios $P^{*} / P_{C}$ and $T_{g} / T_{C}$ are not available for the model. We assume that the corresponding $\mathrm{H}_{2} \mathrm{O}$ values are valid also for the model, and we use these values to estimate $P^{*}$ and $T_{g}$, respectively, for the model. Temperatures are measured in $\mathrm{K}$ for $\mathrm{H}_{2} \mathrm{O}$ and in $\epsilon$ for the model. Pressures are measured in $\mathrm{MPa}$ for $\mathrm{H}_{2} \mathrm{O}$ and in $\epsilon / v_{0}$ for the model.

\begin{tabular}{|c|c|c|c|c|c|c|c|c|}
\hline & $P_{C}$ & $T_{C}$ & $P^{*}$ & $P^{*} / P_{C}$ & $T^{*}$ & $T^{*} / T_{C}$ & $T_{g}$ & $T_{g} / T_{C}$ \\
\hline $\mathrm{H}_{2} \mathrm{O}$ & 22.064 & 647.14 & 0.10133 (Ref. [35]) & $4.6 \times 10^{-4}$ & 277 (Ref. [35]) & 0.428 & 136 (Ref. [36]) & 0.21 \\
\hline Model & $0.6 \pm 0.1$ & $2.0 \pm 0.2$ & $\sim 2.76 \times 10^{-4}$ & & $0.7 \pm 0.1$ & $0.35 \pm 0.06$ & $\sim 0.42$ & \\
\hline
\end{tabular}

The second consideration is related to the possibility that this low- $T$ HDL-LDL phase transition is preempted by inevitable freezing in real water. Recent analysis of the realistic model for water Stillinger-2 (ST2) [15] suggests that the HDL-LDL critical point may occur above the glass temperature $T_{g}$, though as yet still outside the easily accessible experimental range.

We compare our MC results with data for real water. From the location of the liquid-gas critical point $\left(T_{C}, P_{C}\right)$ and the TMD line at ambient pressure $\left(T^{*}, P^{*}\right)$ (Table I), we find the ratios $P^{*} / P_{C}$ and $T^{*} / T_{C}$ in real water. By assuming that the same $P^{*} / P_{C}$ holds in our MC case, we calculate the corresponding $P^{*} \sim\left(2.76 \times 10^{-4}\right)\left(\epsilon / v_{0}\right)$ in our model, and then we estimate the $T^{*}$ corresponding to $P^{*}$ from the TMD line in the MC phase diagram (Fig. 4). In this way, we find a ratio $T^{*} / T_{C}$ from the $\mathrm{MC}$ results, which is consistent with the real water data, suggesting the validity of our assumption on $P^{*} / P_{C}$.

Therefore, we use the same kind of assumption also to estimate the glass temperature $T_{g}$ for our phase diagram. In particular, from real water data we obtain the ratio $T_{g} / T_{C}$ at ambient pressure and, assuming that it holds also for our model, we estimate $T_{g} / \epsilon \sim 0.42$ at $P v_{0} / \epsilon \sim 2.76 \times 10^{-4}$ for the MC phase diagram. Hence, for our model with the parameters chosen in this paper, is $T_{g}>T_{C^{\prime}}$, i.e., the HDLLDL critical temperature at $P_{C^{\prime}} \simeq 0.841 \epsilon / v_{0}$ is below the glass temperature at $P^{*} \sim\left(2.76 \times 10^{-4}\right)\left(\epsilon / v_{0}\right)$. From the study of the phase diagram of real water [4], it is reasonable to expect that $T_{g}(P)$ decreases for increasing $P$, therefore our analysis does not exclude that $T_{C^{\prime}}$ is above $T_{g}\left(P_{C^{\prime}}\right)$. However, by considering a very large $J_{\sigma}$, such that the HDL-LDL critical pressure is $\sim\left(2.76 \times 10^{-4}\right)\left(\epsilon / v_{0}\right)$, we can compare $T_{C^{\prime}}$ and $T_{g}$ at the same pressure $P^{*}$. Our preliminary results show that the HDL-LDL critical temperature is in this case very close to $T_{g}$.

As a consequence of this analysis, our model supports the possibility that the HDL-LDL critical point is located deep into the supercooled region, below or close to the glass temperature, depending on the value of $J_{\sigma}$. Therefore, the liquid-liquid phase transition could be preempted if our model is representative of the thermodynamic properties of the real system. This result is analogous to what has been proposed for silica [15], another liquid with density anomaly, suggesting that the present model could provide a general theoretical framework for anomalous molecular liquids.

The third consideration is about the role of the tetrahedrality in determining the properties of anomalous liquids. For the present model we do not consider a tetrahedral geometry in the two-dimensional MC approach, and the geometry is not explicitly defined in the mean-field approach. Nevertheless, our results are consistent with the experimentally accessible phase diagram of real water, suggesting that the tetrahedral network is not an essential feature for the anomalous behavior of waterlike liquids.

This conclusion is consistent with what has been observed by Angell in Ref. [7], and is well described by a general cooperative model [6] with a generic drive to phase separate the excitations into distinct regions of space (clustering). In our model the drive is given by the intramolecular interaction that mimics the geometrical drive in tetrahedral liquids even if it is not necessarily limited to the tetrahedral case.

In conclusion, we studied the effect of an intramolecular interaction $J_{\sigma}$ in a model for anomalous molecular liquids with a mean-field approach, valid for $J_{\sigma}>0$, and with an off-lattice MC simulation. For $J_{\sigma}>0$ we found a HDL-LDL phase transition while our MC results confirm that for $J_{\sigma}$ $=0$ the singularity-free scenario holds [22]. Hence, the two interpretations originate from the same mechanism with a different hypothesis on the intramolecular interaction; the latter is strictly valid only for $J_{\sigma}=0$. Within this framework, the most reasonable scenario for water includes a HDL-LDL phase transition, probably hindered by inevitable freezing. Our results also suggest that the tetrahedrality is not essential to understand the anomalous behavior in waterlike liquids.

\section{ACKNOWLEDGMENTS}

We thank C. A. Angell, M. Barbosa, A. Scala, F. Sciortino, and M. Yamada for valuable discussions and the NSF (Grant No. CHE0096892) for support. M. I. Marqués thanks the financial support of the Spanish Ministry of Education.
[1] P.G. Debenedetti, Metastable Liquids: Concepts and Principles (Princeton University Press, Princeton, 1996).

[2] For a review, see New Kinds of Phase Transistions: Transformations in Disordered Substances, Proceedings of the NATO
Advanced Research Workshop, Volga River, edited by V.V. Brazhkin, S.V. Buldyrev, V.N. Ryzhov, and H.E. Stanley (Kluwer Academic Publishers, Dordrecht, 2002).

[3] V.M. Glazov, S.N. Chizhevskaya, and S.B. Evgen'ev, Russ. J. 
Phys. Chem. 43, 201 (1969); F. Spaepen and D. Turnbull, in Laser-Solid Interactions and Laser Processing-1978, edited by S.D. Ferris, H.J. Leamy, and J.M. Poake, AIP Conf. Proc. No. 50 (AIP, New York, 1979), p. 73; B.G. Bagley and H.S. Chen, ibid., 97; I.L. Aptecar, Sov. Phys. Dokl. 24, 993 (1979).

[4] C.A. Angell, S. Borick, and M. Grabow, J. Non-Cryst. Solids 207, 463 (1996).

[5] P.H. Poole, M. Hemmati, and C.A. Angell, Phys. Rev. Lett. 79, 2281 (1997); C.A. Angell, R.D. Bressel, M. Hemmati, E.J. Sare, and J.C. Tucker, Phys. Chem. Chem. Phys. 2, 1559 (2000).

[6] C.A. Angell, J. Phys.: Condens. Matter 12, 6463 (2000); C.A. Angell and C.T. Moynihan, Metall. Mater. Trans. B 31B, 587 (2000); C.A. Angell and R.J. Rao, J. Chem. Phys. 57, 470 (1972)

[7] C.A. Angell, in Physics and Applications of Disordered Materials, edited by M. Popescu (INOE, Bucharest, 2002), Chap. 1, p.1.

[8] P.H. Poole, F. Sciortino, T. Grande, H.E. Stanley, and C.A. Angell, Phys. Rev. Lett. 73, 1632 (1994).

[9] S.S. Borick, P.G. Debenedetti, and S. Sastry, J. Phys. Chem. 99, 3781 (1995); C.J. Roberts, A.Z. Panagiotopulos, and P.G. Debenedetti, Phys. Rev. Lett. 77, 4386 (1996); C.J. Roberts and P.G. Debenedetti, J. Chem. Phys. 105, 658 (1996); T.M. Truskett, P.G. Debenedetti, S. Sastry, and S. Torquato, ibid. 111, 2647 (1999).

[10] O. Mishima, Phys. Rev. Lett. 85, 334 (2000); O. Mishima and Y. Suzuki, Nature (London) 419, 599 (2002); O. Mishima and H. E. Stanley, Nature (London) 396, 329 (1998).

[11] A.K. Soper and M.A. Ricci, Phys. Rev. Lett. 84, 2881 (2000); J.L. Finney, A. Hallbrucker, I. Kohl, A.K. Soper, and D.T. Bowron, ibid. 88, 225503 (2002).

[12] J.N. Glosli and F.H. Ree, Phys. Rev. Lett. 82, 4659 (1999).

[13] Y. Katayama, T. Mizutani, W. Utsumi, O. Shimomura, M. Yamakata, and K. Funakoshi, Nature (London) 403, 170 (2000); Y. Katayama, K. Tsuji, H. Kanda, H. Nosaka, K. Yaoita, T. Kikegawa, and O. Shimomura, J. Non-Cryst. Solids 207, 451 (1996).

[14] T. Morishita, Phys. Rev. Lett. 87, 105701 (2001).

[15] I. Saika-Voivod, F. Sciortino, and P.H. Poole, Phys. Rev. E 63, 011202 (2000).

[16] G. Franzese, G. Malescio, A. Skibinsky, S.V. Buldyrev, and H.E. Stanley, Nature (London) 409, 692 (2001); Phys. Rev. E 66, 051206 (2002).
[17] H.K. Lee and R.H. Swendsen, Phys. Rev. B 64, 214102 (2001).

[18] N. Guisoni and V.B. Henriques, J. Chem. Phys. 115, 5238 (2001).

[19] R.J. Speedy, J. Phys. Chem. 86, 3002 (1982); M.C. D’Antonio and P.G. Debenedetti, J. Chem. Phys. 86, 2229 (1987).

[20] S. Sastry, F. Sciortino, and H.E. Stanley, J. Chem. Phys. 98, 9863 (1993).

[21] H.E. Stanley and J. Teixeira, J. Chem. Phys. 73, 3404 (1980).

[22] S. Sastry, P.G. Debenedetti, F. Sciortino, and H.E. Stanley, Phys. Rev. E 53, 6144 (1996); L.P.N. Rebelo, P.G. Debenedetti, and S. Sastry, J. Chem. Phys. 109, 626 (1998); E. La Nave, S. Sastry, F. Sciortino, and P. Tartaglia, Phys. Rev. E 59, 6348 (1999).

[23] P.H. Poole, F. Sciortino, U. Essman, and H.E. Stanley, Nature (London) 360, 324 (1992).

[24] C.W. Kern and M. Karplus, in Water: A Comprehensive Treatise, edited by F. Franks (Plenum Press, New York, 1972), Vol. 1, pp. 21-91; J.B. Hasted, ibid., pp. 255-309; K. Ichikawa, Y. Kameda, T. Yamaguchi, H. Wakita, and M. Misawa, Mol. Phys. 73, 79 (1991).

[25] P.L. Silvestrelli and M. Parrinello, J. Chem. Phys. 111, 3572 (1999).

[26] P.A. Netz, F. Starr, M.C. Barbosa, and H.E. Stanley, Physica A 314, 470 (2002).

[27] G. Franzese, M. Yamada, and H.E. Stanley, in Statistical Physics, edited by M. Tokuyama and H. E. Stanley (AIP, Melville, NY, 2000), pp. 281-288.

[28] G. Franzese and H.E. Stanley, J. Phys.: Condens. Matter 14, 2201 (2002).

[29] This choice turns out reasonable, since the MC results agree with the mean-field predictions, expected to be valid for large dimensions.

[30] M.S.S. Challa, D.P. Landau, and K. Binder, Phys. Rev. B 34, 1841 (1986).

[31] N.B. Wilding and K. Binder, Physica A 231, 439 (1996).

[32] A.M. Ferrenberg and R.H. Swendsen, Phys. Rev. Lett. 61, 2635 (1988).

[33] See Ref. [55] in Ref. [15].

[34] Note that our mean-field approach is valid only for the case $J_{\sigma}>0$, because it assumes the symmetry breaking for the Potts variables. For $J_{\sigma}=0$, the valid mean-field approach is the one presented in Ref. [22], which gives rise to the singularity-free interpretation.

[35] R.A. Fine and F.J. Millero, J. Chem. Phys. 59, 5529 (1973).

[36] G.P. Joharim, A. Hallbrucher, and E. Mayer, Nature (London) 330, 552 (1987) 